\title{
Immunologic Studies in Human Organ Transplantation. III. The Relationship of Delayed Cutaneous Hy- persensitivity to the Onset of Attempted Kidney Allograft Rejection*
}

\author{
W. E. C. Wilson, † Charles H. Kirkpatrick, \\ (From the Department of Medicine, University of Colorado Medical Center, Denver, Colo.)
}

The results of a number of investigations indicate that one feature of advanced renal failure is impaired ability to reject allogeneous tissue grafts. In 1955, Hume, Merrill, Miller, and Thorn reported the transplantation of kidneys from unrelated donors to nine patients with terminal renal failure (1). Four of the nine kidneys functioned for 5 to 25 weeks even though the therapy given to prevent graft rejection was inadequate by current standards. In a study of six uremic patients, Dammin, Couch, and Murray reported histological evidence of skin allograft survival for as long as 115 days (2). In view of these reports, and because a large number of patients were being evaluated for kidney transplantation, we have undertaken a detailed study of the effect of irreversible kidney failure on a number of parameters of immunological capability. Our earlier studies (3) indicated that chronically uremic patients have a suppression of immediate and delayed cutaneous hypersensitivity.

Many features of delayed hypersensitivity are also characteristics of the allograft rejection phenomenon. In both reactions, histologic examination reveals a predominantly lymphocytic infiltration. The two responses can be passively transferred by immunologically competent cells, but serum from an immune animal will not sensitize a nonimmune recipient. Extracts of human peripheral blood leukocytes transfer delayed hypersen-

* Submitted for publication April 6, 1964; accepted June 12, 1964.

Supported by U. S. Public Health Service research grant no. AI-04152.

$\dagger$ Research fellow of the American College of Physicians, 1962-65. Present address: Department of Medicine, Queen's University, Kingston, Ontario, Canada.

¥Special postdoctoral fellow, U. S. Public Health Service. sitivity, and extracts of immunologically competent cells of immune animals will enhance allograft rejection by nonimmune animals $(4,5)$. Because of the association between these two immunological reactions under experimental conditions, it seemed important to define their interrelationship as it applies to human kidney transplantation. The degree of suppression of delayed cutaneous hypersensitivity observed in a group of patients with advanced renal failure has been compared to the response of the group to allogeneous kidney grafts. Patients who demonstrated the most profound impairment of delayed cutaneous reactivity were also found to have the longest intervals between transplantation and the onset of the first immunologic reaction.

\section{Methods}

The subjects of this investigation were 26 patients with chronic renal insufficiency who were accepted for kidney transplantation. In most instances the uremia was the result of chronic glomerulonephritis. Three patients had polycystic kidney disease; two patients had chronic pyelonephritis; one patient had gouty nephropathy. Only a few patients had had renal biopsy in the earlier stages of their disease; however, the histologic nature of the renal lesion was characterized in all patients when their kidneys were removed in preparation for transplantation. The group consisted of 20 males and 6 females whose ages ranged from 6 to 54 years. The mean age was 30.8 years. The pertinent clinical features of the patients are summarized in Table I. The comparatively low values for blood urea nitrogen listed for some patients were obtained after hemodialyses. The values for endogenous creatinine clearance recorded for 19 patients document the marked reduction in functional renal mass that existed in these patients.

The ability of each patient in the group to manifest delayed cutaneous hypersensitivity was assessed before surgery and the administration of any drugs that are known to alter this immunologic reaction. For this evaluation, the cutaneous response to a panel of seven 
TABLE I

Clinical features of the uremic patients*

\begin{tabular}{|c|c|c|c|c|c|c|c|c|c|}
\hline Patients & Sex & Age & Diagnosis & BUN & $\mathrm{C}_{\mathrm{Cr}}$ & $\begin{array}{l}\text { Donor } \\
\text { relationship }\end{array}$ & $\begin{array}{l}\text { Ische- } \\
\text { mia }\end{array}$ & $\begin{array}{c}\text { Reac- } \\
\text { tion } \\
\text { time }\end{array}$ & $\begin{array}{c}\text { Survival } \\
\text { after } \\
\text { transplan- } \\
\text { tationt }\end{array}$ \\
\hline \multicolumn{10}{|l|}{ Responsive } \\
\hline 1. S.H. & $\mathbf{M}$ & 43 & $\mathrm{CGN}$ & 118 & 2.7 & Wife & 38 & $\mathbf{0}$ & $230+$ \\
\hline 2. L.C. & $\mathbf{M}$ & 46 & $\mathrm{CGN}$ & 178 & 2.2 & Sister & 84 & $\mathbf{0}$ & 10 \\
\hline 3. L.S. & $\mathbf{M}$ & 6 & CGN & 53 & 8.8 & Mother & 41 & 1 & 202 \\
\hline 4. R.W. & M & 42 & Gout-neph. & 190 & N.D. & None & 23 & 2 & 36 \\
\hline 5. T.G. & $\mathbf{M}$ & 21 & $\mathrm{CPN}$ & 138 & N.D. & Mother & 30 & 3 & 48 \\
\hline 6. H.O. & $\mathbf{M}$ & 41 & CGN & 116 & N.D. & Wife & 41 & 4 & $305+$ \\
\hline 7. H.M. & $\mathbf{M}$ & 54 & $\mathrm{CGN}$ & 204 & 3.1 & None & 42 & 5 & 14 \\
\hline 8. A.C. & $\mathrm{F}$ & 10 & CGN & 137 & 1.1 & Mother & 42 & 5 & $233+$ \\
\hline 9. P.H. & $\mathbf{M}$ & 40 & $\mathrm{CGN}$ & 166 & N.D. & None & 30 & 6 & $244+$ \\
\hline 10. B.W. & $\mathbf{M}$ & 36 & CGN & 180 & 2.3 & None & 19 & 6 & 21 \\
\hline 11. T.S. & $\mathbf{M}$ & 20 & CGN & 132 & 0.2 & Mother & 24 & 9 & $226+$ \\
\hline 12. D.R. & $\mathbf{M}$ & 39 & CGN & 38 & 5.7 & Sister & 22 & 9 & $312+$ \\
\hline \multicolumn{10}{|c|}{ Unresponsive } \\
\hline 13. L.L. & $\mathbf{M}$ & 22 & $\mathrm{CGN}$ & 140 & 1.7 & Brother & 22 & 2 & $328+$ \\
\hline 14. N.W. & $\mathrm{F}$ & 18 & $\mathrm{CGN}$ & 118 & 1.8 & Mother & 29 & 5 & $237+$ \\
\hline 15. L.C. & $\mathrm{F}$ & 44 & CGN & 116 & N.D. & Brother & 23 & 7 & 83 \\
\hline 16. C.M. & $\mathbf{M}$ & 47 & CGN & 41 & 0.04 & Brother & 40 & 7 & $359+$ \\
\hline 17. T.E. & $\mathbf{M}$ & 16 & Polycystic & 99 & 3.7 & None & 26 & 10 & 95 \\
\hline 18. M.H. & $\mathbf{M}$ & 21 & CGN & 116 & 1.3 & Brother & 33 & 12 & 42 \\
\hline 19. J.H. & $\mathbf{M}$ & 49 & CGN & 132 & N.D. & Sister & 34 & 14 & 24 \\
\hline 20. M.C. & $\mathrm{F}$ & 19 & CGN & 172 & 1.6 & None & 21 & 14 & $271+$ \\
\hline 21. R.C. & $\mathbf{M}$ & 46 & Polycystic & 140 & 1.9 & None & 43 & 15 & $279+$ \\
\hline 22. M.S. & $\mathbf{M}$ & 15 & CGN & 122 & 0.3 & Mother & 26 & 20 & $333+$ \\
\hline 23. S.W. & $\mathrm{F}$ & 15 & Polycystic & 116 & 4.0 & Mother & 37 & 21 & $293+$ \\
\hline 24. L.C. & $\mathbf{M}$ & 42 & $\mathrm{CPN}$ & 285 & N.D. & Brother & 28 & 22 & $331+$ \\
\hline 25. G.S. & $\mathbf{M}$ & 33 & $\mathrm{CGN}$ & 105 & 1.6 & Brother & 40 & 31 & $284+$ \\
\hline 26. D.S. & $\mathrm{F}$ & 15 & $\mathrm{CGN}$ & 74 & 6.7 & Mother & 18 & 33 & $317+$ \\
\hline
\end{tabular}

* CGN = chronic glomerulonephritis; gout-neph. = gouty nephropathy $; \mathrm{CPN}=$ chronic pyelonephritis; $\mathrm{BUN}=$ blood urea nitrogen; $\mathrm{C}_{\mathrm{Cr}}=$ creatinine clearance; N.D. $=$ not determined.

$\uparrow$ Patients designated + are living June $1,1964$.

antigens expected to elicit delayed cutaneous reactions in sensitive subjects was documented. The antigens used were intermediate strength purified protein derivative (PPD), histoplasmin, blastomycin, coccidioidin, mumps skin test antigen, and purified extracts of Candida albicans and Trichophyton inguinale. These antigens were prepared and administered as previously described, and an induration of $0.5 \mathrm{~cm}$ in diameter or greater was considered to be a positive response (3).

In order to ascertain the incidence of delayed cutaneous reactivity in comparable normal subjects, the same antigens were administered intradermally to the prospective donors of kidneys for these uremic patients. In 19 instances the donor and recipient were genetically related, and in three additional cases the prospective donors were the patients' wives. The normal group consisted of 11 males and 15 females whose ages ranged from 21 to 56 years, with a mean age of 37.4 years. Although three of these normal subjects were not accepted as donors, they were used for the comparison of delayed hypersensitivity reactions because of their genetic or environmental relationship. Unrelated volunteer kidney donors were substituted for these three individuals.

The kidney transplantation was performed on 26 donorrecipient pairs of whom 17 were genetically related.
In each instance a living donor was used. With the exception of Patient 3 , the $\mathrm{ABO}$ blood group antigens of the donors were compatible with those of their recipients. The erythrocyte type of Patient 3 was $O$, and he received a kidney from his mother whose type was $\mathrm{A}$. His postoperative course is discussed in detail below.

At the conclusion of the preoperative studies, treatment with azathioprine ${ }^{1}$ was begun. The dose was usually 3 to $5 \mathrm{mg}$ per $\mathrm{kg}$ per day. The patients were then subjected to splenectomy, bilateral nephrectomy, and renal transplantation as described by Starzl, Marchioro, and Waddell (6). The duration of ischemia of the transplanted kidney was recorded at each operation. During the postoperative period the azathioprine treatment was continued. When attempted allograft rejection became apparent, prednisone and actinomycin $\mathrm{C}^{2}$ were added to the therapeutic regime.

The features that characterize the attempted rejection of a kidney allograft are fever, leukocytosis, fall in urine volume, decrease in endogenous creatinine clearance, decrease in urinary sodium excretion, and rise in blood

\footnotetext{
1 Imuran, Burroughs Wellcome \& Co., Tuckahoe, N. Y. 2 Sanamycin, Farbenfibriken Bayer AG, Leverkusen, Germany.
} 
urea nitrogen. A favorable response to treatment for attempted rejection supports the diagnosis. In this study the reaction time is defined as the number of days that elapsed after transplantation before these changes in status appeared and the therapy for reversal of the attempted rejection was begun. Figure 1 summarizes the postoperative course of Patient 22 whose rejection reaction was typical of that observed in the majority of the patients. His reaction time was recorded as 20 days. Unusual patterns of attempted rejection were suspected in Patients 3 and 24, and their postoperative courses are discussed below.

To define the relationship between suppression of delayed cutaneous hypersensitivity and attempted rejection of the kidney allograft, the recipients have been divided into two groups. Those patients who developed no reactions to the antigens have been designated the unresponsive group. The remaining patients, the responsive group, were those in whom a delayed reaction to at least one antigen appeared. The mean reaction time of each group was calculated. The groups were also compared with respect to their mean ages and to the mean duration of ischemia of the transplanted kidneys. The influence of the genetic relationship of the donor and recipient on the reaction time was also evaluated.

\section{Results}

The results of an earlier study indicated that delayed cutaneous hypersensitivity was suppressed in the chronically uremic patient. Because this observation has been reported in detail (3), the results in these 26 patients will be summarized only briefly here. Figure 2 illustrates the percentage of the group in whom reactions to each of the antigens were observed. Blastomycin is not included in the figure because no reactions to this antigen were observed in either the patient or the normal group. In the uremic subjects a total of 167 skin tests was performed, and 21 positive reactions were observed. In the 12 responsive recipients, 1.8 positive tests per patient were observed.

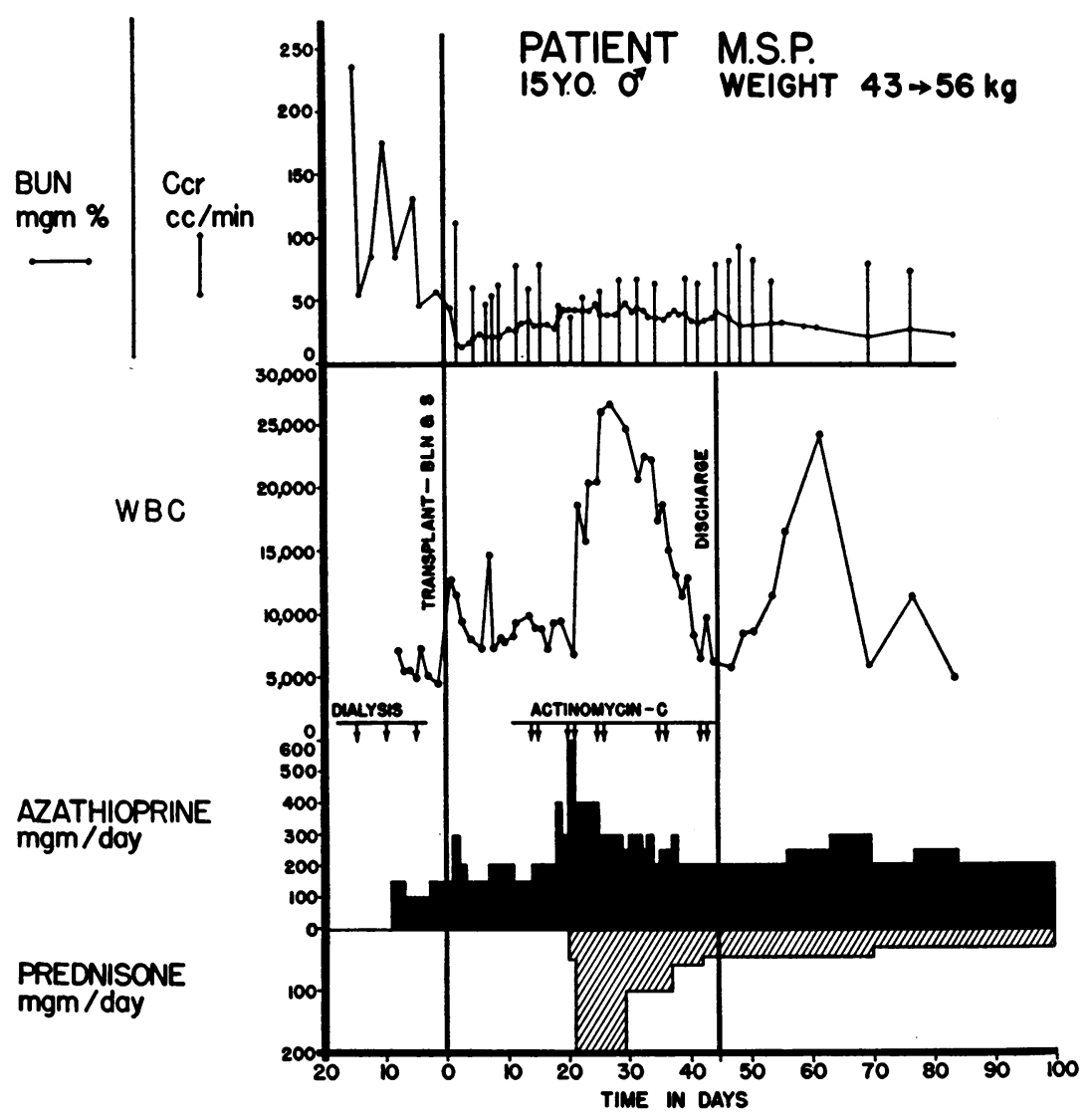

Fig. 1. Clinical course of Patient 22 in whom the diagnosis of attempted REJection Was made on tHe twentieth postoperative day. Note the leukocytosis, rise in blood urea nitrogen (BUN), and fall in endogenous creatinine clearance $\left(\mathrm{C}_{\mathrm{Cr}}\right)$ associated with the immunologic reaction. 


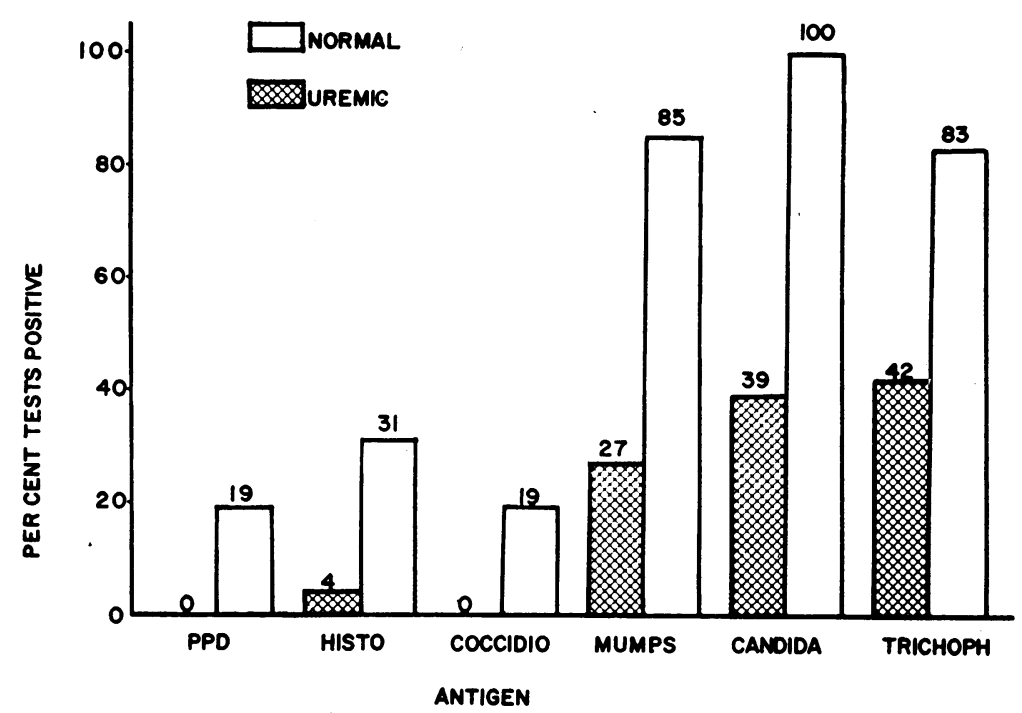

Fig. 2. InCidence of positive delayed hypersensitivity Reactions in NORMAL AND UREMIC SUBJECTS. PPD = purified protein derivative; histo $=$ histoplasmin $;$ coccidio $=$ coccidioidin $;$ Candida $=$ Candida albicans; Trichoph $=$ Trichophyton inguinale.

The responses of the prospective donor group are also summarized in Figure 2. The group was tested 167 times, and 73 positive reactions were recorded. All but one normal subject reacted to at least one antigen, and the mean frequency was 2.8 reactions per person.

The relationships between the responsive and unresponsive recipients with respect to their mean reaction times, mean ages, and the mean ischemia times of the transplanted kidneys are summarized in Table II. The reaction times in the group of 14 unresponsive recipients varied from 2 to 33 days. The mean reaction time of this group was 15.2 days. The responsive recipients manifested episodes of attempted rejection from the day of surgery to 9 days after transplantation, and the mean reaction time observed in this group was 4.2 days. The ages of the unresponsive recipients ranged from 15 to 49 years. Their mean age was

TABLE II

Comparison of means of reaction times, ischemia times, and ages of all patients

\begin{tabular}{lccc}
\hline \hline Group & $\begin{array}{c}\text { Reaction } \\
\text { time }\end{array}$ & $\begin{array}{c}\text { Ischemia } \\
\text { time }\end{array}$ & Age \\
\hline & days & min & $y r s$ \\
Responsive & 4.2 & 36.3 & 33.2 \\
Unresponsive & 15.2 & 30.0 & 28.7 \\
p & $<0.001$ & $>0.20$ & $>0.40$ \\
\hline
\end{tabular}

28.7 years. The responsive recipients were from 6 to 54 years of age, with a mean age of 33.2 years.

The ischemia times of kidneys transplanted to unresponsive recipients varied from 18 to $43 \mathrm{~min}$ utes, and the mean ischemia time of this group was 30.0 minutes. The responsive recipients were transplanted with kidneys that had been subjected to 19 to 84 minutes of ischemia. Their mean ischemia time was 36.3 minutes. Patient 2 , a responsive recipient, was remarkable because he received a kidney that was ischemic for 84 minutes, twice as long as any other kidney in this group. However, he was included in the series because attempted rejection may occur within a similarly short time interval in the absence of prolonged ischemia. In addition, the kidney did elaborate a small quantity of urine initially, and kidney function has been reported despite ischemia times of more than 84 minutes. Finally, on histologic examination of the kidney at 48 hours after transplantation, a picture of rejection that consisted of vasculitis and infiltration of the organ with lymphoid cells was present. Excluding this patient, the mean ischemia time of the responsive recipient group was 32.0 minutes.

Eleven of the 14 unresponsive recipients received kidneys from genetically related donors. 
As shown in Table III, the mean reaction time of these unresponsive recipients was 15.8 days. The mean reaction time of the remaining three unresponsive recipients was 13.0 days. Six of the 12 responsive recipients were transplanted with kidneys from genetically related donors, and the mean reaction time of these recipients was 4.5 days. The remaining six responsive patients who received kidneys from unrelated donors manifested attempted rejection at an average of 3.8 days.

The reaction times were also compared after all 26 patients had been divided on the basis of those whose donors were genetically related and those whose donors were not. These data are shown in the lower part of Table III. The reaction times in the group receiving genetically related kidneys varied from 0 to 33 days, and the mean time of response was 11.8 days. The nine patients who received kidneys from unrelated donors attempted rejection beginning from 0 to 15 days after transplantation. Their mean reaction time was 6.9 days.

The donors of kidneys for patients in the unresponsive recipient group were compatible with respect to the $\mathrm{ABO}$ blood group antigen system. For the responsive recipient group, all donors but one (donor for Patient 3) were also compatible for $\mathrm{ABO}$ erythrocyte antigens. Patient 3, a 6year-old Caucasian male, with erythrocyte type $\mathrm{O}$, received a kidney from his mother, whose erythrocyte type was A. After implantation, immediate function of the kidney was observed at the operating table. However, rapid deterioration of function became apparent within 24 hours, and, in association with this deterioration, his titer of anti-A agglutinin fell from $1: 128$ to $1: 4$. Therapy to reverse attempted rejection was instituted immediately. Gradual improvement occurred and by the twentieth postoperative week renal function was normal. The pathogenesis of the reaction observed in this patient is not clear. He was included with the group because successful kidney transplantation has been observed despite the use of a donor of mismatched $\mathrm{ABO}$ type, and the reaction observed in this patient was not characteristic of rejection that has been ascribed primarily to the isohemagglutinins ( 7$)$. In addition, the attempted rejection was reversed. This has not
TABLE III

Comparison of reaction times of the patients receiving kidneys from related and unrelated donors

\begin{tabular}{|c|c|c|c|}
\hline Group & $\begin{array}{c}\text { No. of } \\
\text { patients }\end{array}$ & $\begin{array}{c}\text { Reaction } \\
\text { time }\end{array}$ & $\mathrm{SE}$ \\
\hline \multicolumn{4}{|l|}{ Related donor } \\
\hline $\begin{array}{l}\text { Unresponsive } \\
\text { Responsive } \\
\text { p }\end{array}$ & $\begin{array}{r}11 \\
6\end{array}$ & $\begin{array}{l}15.8 \\
4.5 \\
0.025\end{array}$ & $\begin{array}{l}3.1 \\
1.6\end{array}$ \\
\hline \multicolumn{4}{|l|}{ Unrelated donor } \\
\hline $\begin{array}{l}\text { Unresponsive } \\
\text { Responsive } \\
\text { p }\end{array}$ & $\begin{array}{l}3 \\
6\end{array}$ & $\begin{array}{l}13.0 \\
3.8 \\
0.001\end{array}$ & $\begin{array}{l}1.5 \\
1.0\end{array}$ \\
\hline \multicolumn{4}{|l|}{ All patients } \\
\hline \multicolumn{4}{|l|}{ Related donor } \\
\hline $\begin{array}{l}\text { Parent } \\
\text { Sibling } \\
\text { Total }\end{array}$ & $\begin{array}{r}8 \\
9 \\
17\end{array}$ & $\begin{array}{l}12.1 \\
11.6 \\
11.8\end{array}$ & $\begin{array}{l}4.0 \\
3.4 \\
2.5\end{array}$ \\
\hline Unrelated donor & 9 & 6.9 & 1.8 \\
\hline $\mathrm{p}$ (related vs. & elated) $\mathrm{C}$ & $5>p>$ & \\
\hline
\end{tabular}

been possible in the reported instances of isohemagglutinin-mediated kidney rejection. If he is omitted from the responsive recipient group, the mean reaction time for the group becomes 4.5 days.

In general, the response of the recipients to the transplanted kidneys was similar, varying to an important degree only in the time of onset. In most instances, the changes in the various parameters of clinical and laboratory status outlined above occurred almost simultaneously, and, after review of the patients' clinical records, the onset of attempted rejection could be recognized with ease. Twenty-five of the 26 patients received therapy for reversal of rejection. The postoperative course of the exceptional patient (Patient 24 ) is summarized in Figure 3. This 42-year-old Caucasian male did not have demonstrable delayed cutaneous hypersensitivity to the antigens. A kidney from his 38 -year-old brother was transplanted and it functioned immediately. On postoperative day 22 he developed fever, eosinophilia, and minor changes in endogenous creatinine clearance and blood urea nitrogen values. This syndrome disappeared coincident with the addition of chloramphenicol to his antibiotic treatment. Whether the abnormalities that developed were the result of an unidentified infection or the result of a mild immunologic reaction that reversed itself spontaneously cannot be ascertained. If this 


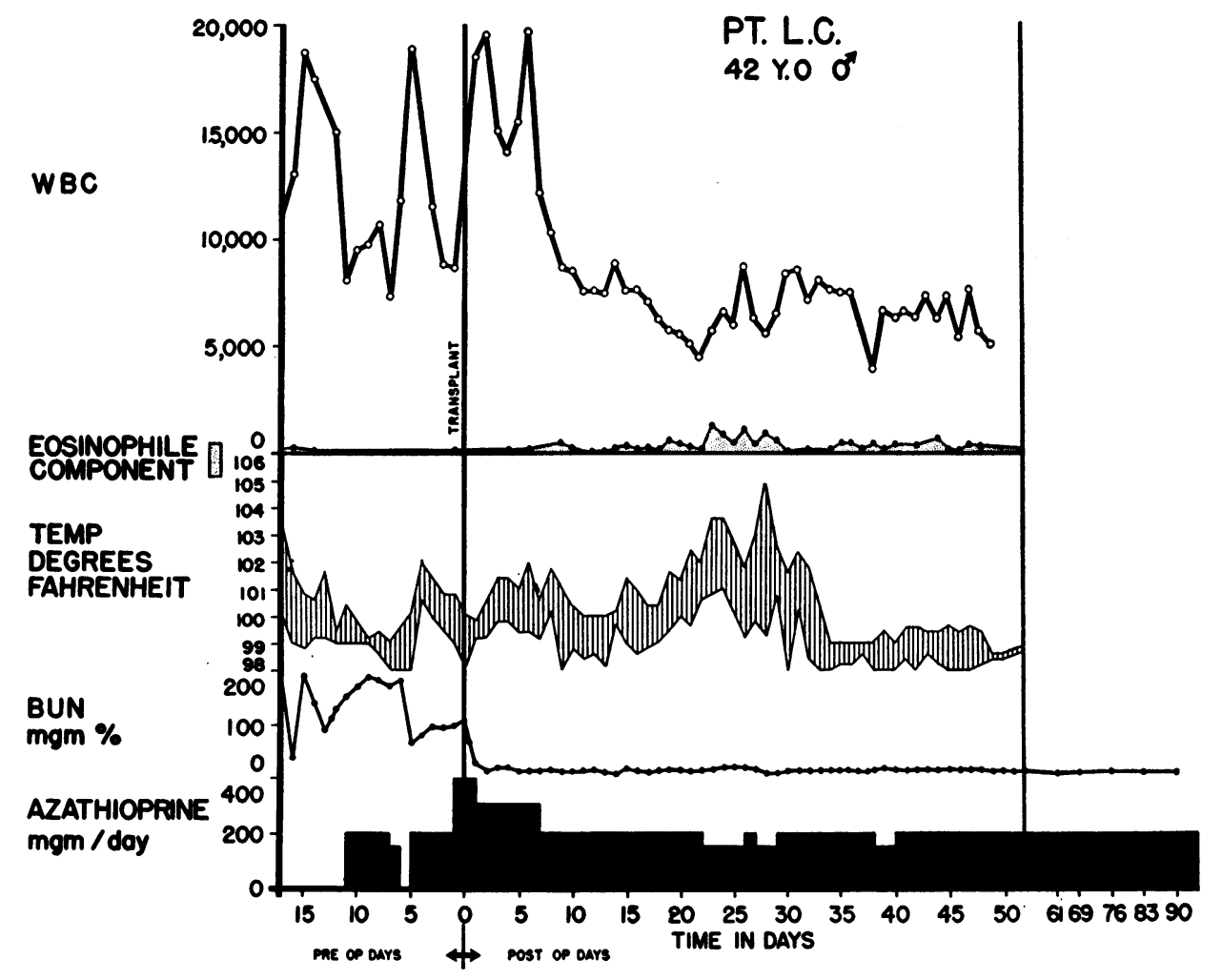

Fig. 3. Clinical course of Patient 24 who developed feVer, leukocytosis, and eosinoPHILIA 22 DAYs After transplantation. Note that only a small rise in BUN was associated with this phenomenon.

patient is deleted from the unresponsive recipient group, the mean reaction time for the group becomes 14.7 days.

Critical review of the 26 patients who received kidney allografts may indicate that three patients constituted exceptions and therefore should not have been included with the remainder of the group. In the unresponsive recipient category, Patient 24 may never have attempted rejection in the 11 months that have elapsed since his transplantation. Among the responsive group, the fail-

TABLE IV

Comparison of means of reaction times, ischemia times, and ages of responsive and unresponsive groups after excluding Patients 2, 3, and 24

\begin{tabular}{cccc}
\hline \hline Group & $\begin{array}{c}\text { Reaction } \\
\text { time }\end{array}$ & $\begin{array}{c}\text { Ischemia } \\
\text { time }\end{array}$ & Age \\
\hline & days & min & yrs \\
Responsive & 4.9 & 31.3 & 24.6 \\
Unresponsive & 14.7 & 30.2 & 27.7 \\
p & $<0.01$ & NS* $^{*}$ & NS* $^{*}$ \\
\hline
\end{tabular}

* Not statistically significant. ure of function of the kidney transplanted to $\mathrm{Pa}$ tient 2 may have been the result of the long duration of ischemia, and the early deterioration of renal function observed in Patient 3 may have been mediated by humoral antibody (anti-A agglutinin). Table IV summarizes the interrelationships of mean reaction times, mean ages, and mean ischemia times when these three patients are excluded. The mean reaction time in the unresponsive group was 14.7 days and in the re-

TABLE V

Survival of recipients 7 months after kidney transplantation

\begin{tabular}{ccc}
\hline & $\begin{array}{c}\text { Responsive } \\
\text { recipients }\end{array}$ & $\begin{array}{c}\text { Unresponsive } \\
\text { recipients }\end{array}$ \\
\hline Related donor & & \\
Parent & $2 / 4$ & $4 / 4$ \\
Sibling & $1 / 2$ & $4 / 7$ \\
Total & $3 / 6$ & $8 / 11$ \\
Unrelated & $3 / 6$ & $2 / 3$ \\
donor & & \\
\hline
\end{tabular}


sponsive group 4.9 days. The mean age of the unresponsive recipients was 27.7 years compared to 34.6 years in the responsive group. In the unresponsive and responsive patients, the mean ischemia times were 30.2 and 31.3 minutes, respectively.

The numbers of patients in each group who are alive at a minimum of 7 months after transplantation are summarized in Table V.

\section{Discussion}

Twenty-seven patients received kidney transplants from 29 donors at the University of Colorado Medical Center between June 27, 1963, and November 9, 1963. During this period, all patients were treated for attempted rejection on the basis of one protocol, namely, that immunosuppressive therapy in addition to azathioprine was not instituted until deterioration of kidney function was clearly apparent. Addition of patients to this study was stopped because of a change in the treatment protocol employed for subsequent recipients. The group comprising the present study includes 26 patients who survived the first 24 postoperative hours and who received kidneys that functioned well enough at the time of implantation to be left in place. One patient, who received a kidney from her father, was excluded from the study because she died approximately 12 hours postoperatively, and the cause of death was electrolyte imbalance occurring during a diuresis of over $18 \mathrm{~L}$. Two patients included in the study (Patients 17 and 18) each received two kidney transplants. In both instances, the donors of the first kidney were not compatible with the recipients with respect to the $\mathrm{ABO}$ blood group antigens, and function of the kidney ceased within minutes after implantation. The two mismatched kidneys were removed at the time of the first opperations. Since the failure of these kidneys appeared to have been mediated by humoral antibody, their rejection was not considered further in this study. These two patients subsequently received second kidney transplants from unrelated volunteers, and the reaction times in response to these grafts were included in the evaluation of the group. No further exclusions were made in the remaining 26 because the postoperative deterioration of renal function was believed to have been the result of attempted allograft rejection. Because Patients 2, 3, and 24 had unusual features in their postoperative courses, the data have been evaluated both after including and excluding these patients.

Delayed cutaneous hypersensitivity is suppressed in patients with advanced renal failure (3). By all parameters studied, the patients in this investigation had irreversible renal failure. Sixteen of the 19 patients in whom endogenous creatinine clearance was evaluated had values of less than 5 $\mathrm{ml}$ per minute, and in all patients the values were less than $10 \mathrm{ml}$ per minute. In a study of the relationship of impaired creatinine clearance and life expectancy in patients with chronic kidney disease, Tobias, McLaughlin, and Hopper reported that only 2 of 17 patients with clearance values that remained below $10 \mathrm{ml}$ per minute were alive 9 months later (8). The seven patients who were not evaluated with respect to creatinine clearance had levels of blood urea nitrogen above $116 \mathrm{mg}$ per $100 \mathrm{ml}$ at the time of intradermal testing. The histologic appearance of the kidneys substantiated the severe degree of kidney destruction that had occurred.

In a study of delayed cutaneous hypersensitivity to tuberculin in seven uremic subjects, Dammin and co-workers concluded that delayed reactivity was not impaired in patients with chronic renal disease (2). In the present investigation, 14 of the 26 uremic recipients were found to be unreactive to a panel of antigens. Only one of the normal subjects did not react to at least one of the antigens. The remaining 12 uremic patients, in whom delayed cutaneous hypersensitivity was demonstrable, were significantly less reactive to the panel of antigens than the normal group. The incidence of positive responses in the reactive recipients was 1.8 tests per person, whereas the incidence in normal subjects was 2.8 tests per person $(p<0.05)$. These observations indicate that the patients with advanced renal failure have suppressed delayed cutaneous reactivity.

The results of the present investigation also indicate that the reaction time can be related to the suppression of delayed cutaneous reactivity. The 14 patients in whom delayed responsiveness could not be demonstrated had a mean reaction time of 15.2 days. By contrast, the remaining 12 patients with only partial suppression of their ability to 
manifest delayed reactions had a mean reaction time of 4.2 days.

A number of factors in addition to the immunologic capability of the recipient influence the survival of a kidney allograft. In general, elderly patients are less likely to be successful kidney allograft recipients than younger patients (9). The chronically uremic older patient is less able to withstand the rigors of extensive abdominal surgery as well as the marked systemic insult associated with the rejection crisis. Presumably this difference with respect to age is related to the presence of more extensive degenerative vascular and cardiorespiratory disease. The present study indicates that the less favorable prognosis in older recipients is not related to a difference in their immunological response, since the reaction time was not significantly different in those over 35 years and those under 35 years. The mean age of the unresponsive recipient group was also not significantly different from the mean age of the responsive recipients (Table II).

An excessive period of ischemia of a transplanted kidney may jeopardize its survival (9). Although in animal studies the deleterious effect of ischemia is more pronounced on allografts than on autografts, the reaction times cannot be related to the ischemia times of the kidneys transplanted in this study. The reaction time to kidneys subjected to less than 40 minutes ischemia was the same as the reaction time to kidneys ischemic for more than 40 minutes. This inability to relate deterioration of function to ischemia time may indicate that ischemia does not enhance immunologic damage. Probably of more relevance is the fact that there was only one kidney exposed to an inordinately long period of ischemia (Patient 2). Whether this patient is included in the group or not, the ischemia times in the unresponsive and responsive groups are not significantly different (Tables II and IV), indicating that ischemia does not influence the relationship reported between cutaneous sensitivity and attempted rejection.

Although matching of the ABO erythrocyte antigens of the donor with those of the recipient does not insure success of a kidney transplant, the insertion of a kidney from a mismatched donor is associated with a high failure rate (10). Because of the rapidity of onset of the rejection of mismatched kidneys, the failure is believed to be primarily the result of the presence of circulating isoagglutinin. Only one patient (Patient 3 ) received a kidney from a donor mismatched with respect to $\mathrm{ABO}$ antigens. He was considered in this series because his kidney did function well immediately after insertion. However, the relationship of cutaneous reactivity to rejection has also been evaluated excluding this patient because the kidney did fail soon after implantation and the failure was associated with a fall in isoagglutinin titer. His exclusion from the group did not alter significantly the difference between the responsive and unresponsive groups (Table IV).

Transplantation studies with laboratory animals have established the importance of the genetic relationship of the donor and recipient. Tissue allografts survive longer when transplanted between mice of strains that share major histocompatibility antigens than when they are exchanged between mice of strains that do not (11). In addition, tolerance of tissue allografts is more easily induced when strains share major transplantation antigens (12).

With respect to the present group of patients, the interval between transplantation and the immunologic response to the kidney did not correlate with the genetic relationship of the donor to the degree that would be expected from the studies with inbred mice. The mean reaction time observed in those recipients whose donors were related was longer than the mean observed in those whose donors were not (11.8 days vs. 6.9 days). However, this difference was not significant $(p=>0.2)$. A poor correlation remained after exclusion of Patients 2 and $3(p=>0.1)$. These results are not interpreted to indicate that the genetic relationship of the donor is a factor of little importance in determining the response of the recipient during the early postoperative period. However, the results do indicate that another variable must exert an effect in this particular clinical model. Age was not a factor in this series of patients because the mean age of the group who received kidneys from related donors was less than the mean age of the group who did not. The mean ischemia times of the two groups were identical, including Patient 2 in the related donor group. 
When the kidney recipients were divided on the basis of the degree of suppression of delayed cutaneous hypersensitivity, a high degree of correlation with the interval between transplantation and response to the kidney was observed (Tables II and IV). The correlation was much better than the correlation observed between the genetic relationship of the donor and the reaction time. This fact is interpreted to indicate that uremic suppression of delayed responsiveness is a factor which appears to supersede in importance the genetic relationship of the donor in determining the recipient's response to this graft during the early postoperative period.

The responsive and unresponsive categories were subdivided on the basis of the genetic relationship of the kidney donor. An attempt was made to evaluate the effect of uremic suppression of delayed responsiveness within the group whose kidney donors were related and also within the group whose donors were not (Table III). A better correlation was observed within the unrelated donor group $(\mathrm{p}=<0.001)$ than within the related donor group $(p=0.025)$. When Cases 2 and 3 were excluded from the related donor group, the numbers of patients were too small to demonstrate a significant correlation $(0.1>\mathrm{p}>0.05)$. Because the numbers of patients are relatively small, conclusions must remain tentative, but apparently the suppressive effect of uremia is more easily demonstrated in the patients who receive kidneys from unrelated donors.

Two additional factors influence the response of laboratory animals and patients to allogeneous tissue grafts. Previous exposure to antigens of a graft may accelerate its rejection. Two patients in the unresponsive recipient group each received two kidney transplants. The reaction times following insertion of the second kidneys were well within the range for the total group of unresponsive recipients. As well as can be determined, all patients were subjected to the same probability of sensitization from leukocytes in transfused blood.

The remaining factor is the degree of immunosuppression induced by chemotherapy. This is difficult to quantitate. Azathioprine was administered on a weight basis initially, and deviations from this dose were dictated by the peripheral blood counts. The aim of treatment was to keep the peripheral leukocyte count in the low normal range. Within this framework all patients received a similar amount of cytotoxic therapy.

With all the variables that are known to influence the survival of tissue allografts considered and those patients who conceivably could have constituted exceptions excluded, it appears clear that the chronically uremic patients with the most profound suppression of delayed cutaneous hypersensitivity manifest attempted rejection later than those patients with less suppression. Patients with Hodgkin's disease also have impaired delayed cutaneous reactivity and have impaired ability to reject skin allografts. In a study that compared these two abnormalities in patients with Hodgkin's disease, skin graft survival was most prolonged in the patients with complete suppression of delayed cutaneous responsiveness (13). The pathogenesis of impaired immunologic capability has not been completely characterized in either chronic uremia or Hodgkin's disease. The mechanism does not appear to be the same in the two diseases. Delayed cutaneous reactivity cannot be acquired by patients with Hodgkin's disease through passive transfer of immunologically competent cells (13). Chronically uremic patients, however, passively acquire delayed cutaneous reactivity as a consequence of the transfer of immunologically competent cells from kidney donors (3).

A relationship between the ability of the chronically uremic patient to manifest delayed cutaneous hypersensitivity and his ability to respond to a transplanted kidney raises a number of important implications. First, the correlation of impaired delayed cutaneous reactivity with the reaction time suggests that the deterioration in renal function that occurred postoperatively in these patients was immunologically mediated, and not the result of failure of vascular anastomoses or other technical factors. Although graft rejection is clearly an immunologic phenomenon, the exclusion of other causes of abrupt deterioration in kidney function in the postoperative period is frequently a difficult clinical problem.

In a number of laboratory models, the successful induction of tolerance depends upon the degree of immune suppression at the time that antigen is 
introduced as a tolerance-conferring stimulus. To determine the possible role of uremic suppression of immunologic responsiveness in the ultimate fate of human kidney transplants, the overall survival of this group of patients was examined. A minimum of 7 months has elapsed since transplantation. Although the primary cause of death was not the result of renal failure following graft rejection, the immunologic response to the kidney may be considered to have been a frequent underlying cause of death. In general, patients with the most intensive immunologic response receive more immunosuppressive therapy, and hence are more subject to fatal infections and bone marrow depression. Ten of 14 unresponsive recipients and 5 of 12 responsive recipients are alive at present. These differences are not significant. In addition, a comparison of the unresponsive group to the responsive group is difficult to interpret because of the preponderance of patients with related donors in the unresponsive group. The genetic relationship of the donor has been established to be an important factor in the long term survival of the human kidney allograft recipient.

Survival data with subdivision of the groups on the basis of donor genetic relationship are summarized in Table V. In each category the unresponsive recipients appear to have a better prognosis, but the differences are not statistically significant. However, it should not be concluded that suppression of the recipient's immunologic responsiveness as a consequence of advanced renal failure has no effect on the ultimate fate of the graft. The majority of patients appeared to have some suppression of delayed cutaneous hypersensitivity, and in this study an attempt has been made to distinguish between two degrees of suppression.

A final implication of the present observations is that they provide a method for the identification of those patients in whom difficulty can be anticipated in the early postoperative period. Adrenal steroid therapy will suppress delayed cutaneous reactivity and its value in the treatment of the rejection crisis has been recently reviewed (14). We might expect that, if the treatment given to the present group of patients to reverse rejection had been instituted in the responsive recipients at the time of transplantation, the immunologic response during the postoperative period would have been delayed. The suppressive influence of the steroid could be provided early in the evolution of the rejection reaction rather than after the immunologic reaction had become well established. This concept is under evaluation in a group of patients currently receiving allogeneous kidney grafts.

\section{Summary}

Twenty-six patients with chronic uremia were studied with respect to their ability to demonstrate delayed cutaneous hypersensitivity reactions to a panel of antigens. All of the patients subsequently received kidney allografts from living donors. Those patients in whom delayed hypersensitivity reactions could be demonstrated, the responsive group, manifested evidence of attempted allograft rejection significantly earlier than those patients with no reactions, the unresponsive group (4.2 days vs. 15.2 days). These differences were independent of the duration of ischemia of the transplanted kidney, the age of the patient, and the relationship of the donor to the recipient. These observations suggest that the chronically uremic patient with the most profound reduction in delayed cutaneous responsiveness is also less able to respond to a kidney allograft. The clinical implications of these observations are discussed.

\section{Acknowledgments}

We thank Drs. T. E. Starzl, T. L. Marchioro, W. R. Waddell, J. H. Holmes, and M. P. Hutt for permission to study their patients.

\section{References}

1. Hume, D. M., J. P. Merrill, B. F. Miller, and G. W. Thorn. Experiences with renal homotransplantation in the human: report of nine cases. J. clin. Invest. 1955, 34, 327.

2. Dammin, G. J., N. P. Couch, and J. E. Murray. Prolonged survival of skin homografts in uremic patients. Ann. N. Y. Acad. Sci. 1957, 64, 967.

3. Kirkpatrick, C. H., W. E. C. Wilson, and D. W. Talmage. Immunologic studies in human organ transplantation. I. Observation and characterization of suppressed cutaneous reactivity in uremia. J. exp. Med. 1964, 119, 727.

4. Lawrence, H. S. The transfer of hypersensitivity of the delayed type in man in Cellular and Humoral 
Aspects of the Hypersensitive States, H. S. Lawrence, Ed. New York, Hoeber-Harper, 1959, p. 279.

5. Najarian, J. S., and J. D. Feldman. Skin homograft rejection by a humoral agent. Fed. Proc. 1963, 22, 274.

6. Starzl, T. E., T. L. Marchioro, and W. R. Waddell. The reversal of rejection in human renal homografts with subsequent development of homograft tolerance. Surg. Gynec. Obstet. 1963, 117, 385.

7. Starzl, T. E., T. L. Marchioro, J. H. Holmes, G. Hermann, R. S. Brittain, O. H. Stonington, D. W. Talmage, and W. R. Waddell. Renal homografts in patients with major donor-recipient blood group incompatibilities. Surgery 1964, 55, 195.

8. Tobias, G. J., R. F. McLaughlin, Jr., and J. Hopper, Jr. Endogenous creatinine clearance. A valuable clinical test of glomerular filtration and a prognostic guide in chronic renal disease. New Engl. J. Med. 1962, 266, 317.

9. Starzl, T. E., T. L. Marchioro, D. Rifkind, J. H. Holmes, D. T. Rowlands, Jr., and W. R. Waddell.
Factors in successful renal transplantation. Surgery 1964, 56, 296.

10. Dempster, W. J. Reassessment of the anurias after kidney transplantation. Brit. med. J. 1963, 1, 1697.

11. Berrian, J. H., and C. F. McKhann. Strength of histocompatibility genes. Ann. N. Y. Acad. Sci. 1960, 87, 106.

12. Martinez, C., F. Shapiro, and R. A. Good. Induction of immunological tolerance of tissue homografts in adult mice in Mechanisms of Immunological Tolerance, M. Hasek, A. Lengerova, and M. Vojtiskova, Eds. New York, Academic Press, 1962, p. 329.

13. Kelly, W. D., D. L. Lamb, R. L. Varco, and R. A. Good. An investigation of Hodgkin's disease with respect to the problem of homotransplantation. Ann. N. Y. Acad. Sci. 1960, 87, 187.

14. Marchioro, T. L., H. K. Axtell, M. F. LaVia, W. R. Waddell, and T. E. Starzl. The role of adrenocortical steroids in reversing established homograft rejection. Surgery 1964, 55, 412. 